\title{
The feasibility of non-contrast-enhanced zero echo time magnetic resonance angiography for characterization of intracranial atherosclerotic disease
}

\author{
Chao Zhang ${ }^{1 \#}$, Weiqiang Dou ${ }^{2 \#}$, Ke Yu ${ }^{1}$, Yun $\mathrm{Ji}^{1,3}$, Wenliang Wang ${ }^{4}$, Muhammad Umair Sami ${ }^{1}$, Yong \\ Shen $^{5}$, Kai Xu ${ }^{1}$ \\ ${ }^{1}$ Department of Radiology, Affiliated Hospital of Xuzhou Medical University, Xuzhou, China; ${ }^{2}$ GE Healthcare, MR Research China, Beijing, China; \\ ${ }^{3}$ Department of Radiology, Traditional Chinese Medical Hospital of Yixing, Yixing, China; ${ }^{4}$ Department of Interventional Radiology, Affiliated \\ Hospital of Xuzhou Medical University, Xuzhou, China; ${ }^{5}$ GE Healthcare, MR Enhanced Application China, Beijing, China
}

\#These authors contributed equally to this work.

Correspondence to: Kai Xu. Affiliated Hospital of Xuzhou Medical University, No. 99, Huaihai West Road, Xuzhou, China. Email: xzmu_xukai@163.com.

Background: Accurate and non-invasive assessment of intracranial atherosclerotic disease (ICAD) is important because of its effect on treatment planning. The aim of this study is to investigate if zero echo time (zTE) magnetic resonance angiography (zTE-MRA) is feasible in the characterization of ICAD.

Methods: A total of 175 patients with ICAD were recruited. ZTE-MRA and time-of-flight (TOF)MRA sequences were conducted for all participants using a 3T clinical MR system. Forty-one patients also underwent digital subtraction angiography (DSA), and were confirmed to have intracranial arterial stenosis (ICAS). Weighted kappa $(\kappa)$ statistics were used to assess the inter-observer agreement and diagnostic consistency of both zTE- and TOF-MRA, using DSA as a reference. The Wilcoxon signed-rank test was used to evaluate differences in image quality between zTE- and TOF-MRA images. The nonparametric test of multiple paired samples was used to compare the results of vascular stenosis diagnosis between zTE-, TOF-MRA and DSA.

Results: Supported by high inter-observer agreement (weighted $\kappa=0.78$ ), zTE-MRA generated significantly higher scores than TOF-MRA for susceptibility artifact signal (mean: $3.03 \pm 0.98$ vs. $2.72 \pm 1.09$; $\mathrm{P}=0.017$ ) and flow signal in parent artery (mean: $3.63 \pm 0.49$ vs. $3.07 \pm 0.82 ; \mathrm{P}<0.001$ ). Additionally, zTE-MRA showed more robust diagnostic performance than TOF-MRA for patients with ICAD and degree of vascular stenosis $(\mathrm{P}<0.05)$, and was highly consistent with reference DSA images (weighted $\kappa=0.80$ ).

Conclusions: ZTE-MRA has potential for use as a routine clinical method for patients with ICAD.

Keywords: Magnetic resonance angiography; intracranial atherosclerosis; digital subtraction; diagnosis

Submitted May 26, 2020. Accepted for publication Jan 06, 2021.

doi: 10.21037/qims-20-696

View this article at: http://dx.doi.org/10.21037/qims-20-696

\section{Introduction}

Intracranial atherosclerotic disease (ICAD) is one of the most common causes of stroke, leading to $10 \%$ of ischemic strokes globally $(1,2)$, and is expected to result in a serious international burden in the future (3-5). Hence, early diagnosis and accurate evaluation of ICAD is crucial.
Multiple angiography techniques, including computed tomography angiography (CTA), magnetic resonance angiography (MRA), and digital subtraction angiography (DSA), have been applied in the clinic for patients with ICAD to date, to assess the degree of stenosis of intracranial arteries, which is considered an important clinical 
manifestation of ICAD (2).

Of the techniques mentioned above, CTA is a noninvasive approach that can diagnose vascular stenosis and occlusion with high sensitivity (97\%) and specificity (99.5\%), using catheter angiography as a reference standard (2). Moreover, this technique allows rapid image acquisition, thereby reducing the potential for motion artifacts (6); however, the risk from radiation and the need for contrast agent administration limit its wide clinical application. DSA has been recognized as a gold standard for evaluation of intracranial cerebrovascular diseases $(7,8)$. In addition to providing accurate assessment of vessel morphology, DSA can offer dynamic information about blood flow at high temporal resolution (9), which cannot be obtained by CTA or MRA; however, due to its invasiveness and high cost, DSA examination has not been adopted as a routine clinical procedure (10).

Non-contrast MRA does not require radiation or contrast agent and has, therefore, been applied for vascular evaluation in the clinic $(6,11)$. In particular, the conventional MRA technique, time-of-flight (TOF)-MRA, has generated promising results for diagnosis of vascular diseases (1). Nevertheless, TOF-MRA imaging is sensitive to disturbance of intraluminal blood flow, particularly leading to overestimation of intracranial stenosis, or even its misdiagnosis as occlusions (12), which is a crucial factor influencing the high sensitivity and low specificity of TOF-MRA for evaluation of intracranial arterial stenosis (ICAS) (13). Moreover, TOF-MRA imaging is prone to the effects of local field inhomogeneities; for example, those caused by implanted intracranial stents (14).

As an alternative, the zero echo time (zTE) radial arterial spin labeling (ASL) has been used for intracranial aneurysm remnant and in-stent in many studies (15-18). Katsuki et al. (19) use this technique to describe the arteries near the cerebral aneurysm with clip and the peripheral cerebral arteries. In addition, other scholars also employed zTE-MRA for evaluating cerebrovascular disorder in clinic, including cerebral arteriovenous malformation and moyamoya disease (20-22). In essence, zTE-MRA uses a continuous ASL strategy and a zTE radial acquisition readout scheme (23). Both these intrinsic properties separately facilitate the acquisition of MRA images, with inflowing blood labeled with an endogenous contrast agent, and with high signal-to-noise-ratio and less sensitivity to local field inhomogeneities. Given these advantages, we aimed to test whether this relatively novel MRA approach is feasible for diagnosis of patients with ICAD and for more accurate assessment of the extent of vascular stenosis. To investigate this, the zTE-MRA technique was applied to a clinical population with confirmed ICAD in this study. Based on the best of our literature review, this is one of the earliest studies using zTE-MRA for ICAD diagnosis and for vascular stenosis level assessment when set DSA as the reference, aiming to observe the clinical feasibility of zTE technology in clinical applications. The corresponding image quality and diagnostic accuracy for arterial stenosis were systematically evaluated and compared with those acquired by TOF-MRA. In addition, DSA imaging was applied and served as a standard reference.

\section{Methods}

\section{Data cohort}

This study was approved by the local ethical committee of Affiliated Hospital of Xuzhou Medical University. Written informed consent was obtained from all patients prior to MR and DSA experiments, according to the Declaration of Helsinki.

In total, 175 patients ( 63 female, mean age $=67.95 \pm$ 8.12 years; 112 male, mean age $=68.18 \pm 7.96$ years), who met the Chinese diagnosis criteria for cerebral atherosclerosis [2014] (24), were recruited between January 2017 and September 2019, and assessed by zTE- and TOF-MRA. The detailed diagnostic strategies are described in a previous report by $\mathrm{Wu}$ et al. (24). The exclusion criteria for participants were as follows: (I) MRI confirmed brain abnormality, including trauma, infection, aneurysm, or moyamoya disease; (II) MRI contraindications, including claustrophobia, implanted metal parts, and ferromagnetic foreign bodies; (III) patients who had undergone internal carotid balloon remodeling or received stent assistance.

Among participants, 41 patients who underwent DSA examination $24 \mathrm{~h}$ after MRA assessment were eligible for enrollment (18 females, mean age $=60.1 \pm 4.43$ years; 23 males, mean age $=60.7 \pm 4.70$ years). The inclusion criteria for DSA examination were as follows: (I) patients with severe stenosis with indication for stent therapy; (II) significant clinical symptoms that did not match the degree of stenosis (e.g., mild and moderate); (III) patients with no contraindications for DSA examination.

\section{MR experiments}

All MRI experiments were performed using a 3T MR 
system (Discovery 750w, GE Healthcare, USA), with a 24-channel head and neck joint coil.

For zTE-MRA measurement, scan parameters included: field-of-view (FOV), $200 \times 200 \mathrm{~mm}$, for parietal to mandible coverage; flip angle $(\mathrm{FA})=3^{\circ}$; matrix size $=166 \times 166$; repetition time $(\mathrm{TR})=1,157 \mathrm{~ms}$; echo time $(\mathrm{TE})=16 \mu \mathrm{s}$; number of slices $=332$; and slice thickness $=0.6 \mathrm{~mm}$. $\mathrm{zTE}$ MRA is in essential a 3D MRA technique with a centerout radial rotating ultrafast imaging sequence (RUFIS) readout applied (25). Inherited from 3D pCASL technique, the labeling plane of zTE-MRA is automatically set $20 \mathrm{~mm}$ beneath the bottom line of the prescribed FOV. The actual TR for 512 radial spokes per segment is $1,157 \mathrm{~ms}$. Therefore, for each spoke acquisition, the time is $1.28 \mathrm{~ms}$. The scan time was 6 min $51 \mathrm{~s}$.

The TOF-MRA sequence was also applied to cover the mesencephalon to cingulate cortex area, with FOV 220x $220 \mathrm{~mm}$. Other scan parameters were: matrix size $=512 \times 192$ without interpolation, $\mathrm{FA}=15^{\circ}, \mathrm{TR}=20 \mathrm{~ms}, \mathrm{TE}=3.4 \mathrm{~ms}$, number of slices $=172$, slice thickness $=0.5 \mathrm{~mm}$ and number of slab $=3$, slab thickness $=46 \mathrm{~mm}$. The acquisition time was $4 \min 41 \mathrm{~s}$.

\section{$D S A$}

DSA was performed using an angiographic unit (Allura Xper FD20, Philips Medical Systems, Amsterdam, Netherlands) by an interventional neuroradiologist (with 14 years of experience). A 4 F Headhunter catheter was introduced into the ascending aorta via the transfemoral route and navigated into the appropriate carotid or vertebral artery, as determined by the neurointerventionist. For the internal carotid artery (ICA), $7 \mathrm{~mL}$ of non-ionic contrast agent (Ioversol, Hengrui Medicine, Jiangsu, China) was injected at a volume rate of $4 \mathrm{~mL} / \mathrm{s}$. For the vertebral artery, $6 \mathrm{~mL}$ was injected at a volume rate of $3 \mathrm{~mL} / \mathrm{s}$. The image matrix and FOV were set separately as $1,024 \times 1,024$ and $170 \times 170 \mathrm{~mm}^{2}$, respectively.

\section{Imaging assessment}

In the current study, both susceptibility artifact intensity and flow signal quality assessment were evaluated. The susceptibility artifact intensity mainly reflected by signal loss, especially in volume rendering (VR) imaging. A 4-point scale $(4=$ no susceptibility signal loss; $3=$ minimal signal loss; $2=$ moderate signal loss, compromising image assessment; and $1=$ severe signal loss, preventing image evaluation), was applied to rate the susceptibility artifact intensity for both zTE- and TOF-MRA images. This method was modified from the 5 -point scale reported by Takano et al. (26) without including the 5 th point (the depiction is almost equal to DSA), since no paired DSA data were acquired. The flow signal is actually the signal intensity and an evaluation of the signal strength uniformity within the lumen. The regions of interest (ROIs) were selected in each interested artery and compared among all subjects measured with different imaging methods. Each ROI had identical area and was prescribed within the blood vessel with a certain distance away from the vessel wall. To rate the flow signal in both zTE- and TOF-MRA images, additional DSA imaging was also available and used as a reference. A separate 4-point scale, reported previously $(15,27)$ for similar analysis, was applied also for data analysis. It is defined as: $4=$ excellent quality, equal to DSA images; $3=$ good quality, with minimal blurring or artifacts; $2=$ poor quality, with structures slightly visible and significant blurring; and $1=$ not visible.

According to the North American Symptomatic Carotid Endarterectomy Test, vascular stenosis was defined as one of four levels, as follows: mild stenosis (luminal stenosis $<50 \%$ ); moderate stenosis (luminal stenosis 50-69\%); severe stenosis (luminal stenosis $\geq 70 \%$ ); and occlusion.

\section{Data analysis}

All acquired zTE- and TOF-MRA images were reconstructed using the maximum intensity projection (MIP), multiple projection reconstruction (MPR), and VR methods of the GE MR workstation (AW 4.6, GE Medical Systems, USA). Two experienced radiologists (each with two years of experience in cerebrovascular disease imaging) were employed to independently analyze the reconstructed images for susceptibility artifact intensity and flow signal as well as DSA images in the arteries of interest. Bilateral ICAs were selected as the main arteries of interest with M1 segment of middle cerebral arteries (MCA) and basilar artery (BA) as the additional arteries of interest for susceptibility artifact intensity and flow signal analysis. The most stenotic area was selected as the ROI for stenosis measurement. Each ROI was measured twice and then averaged as the final result of each reader. As the additional arteries of interest, the detailed results of M1 segment of MCA and BA in terms of susceptibility artifact and flow signal have been shown in Figure S1.

All statistical analyses were performed using SPSS 
A

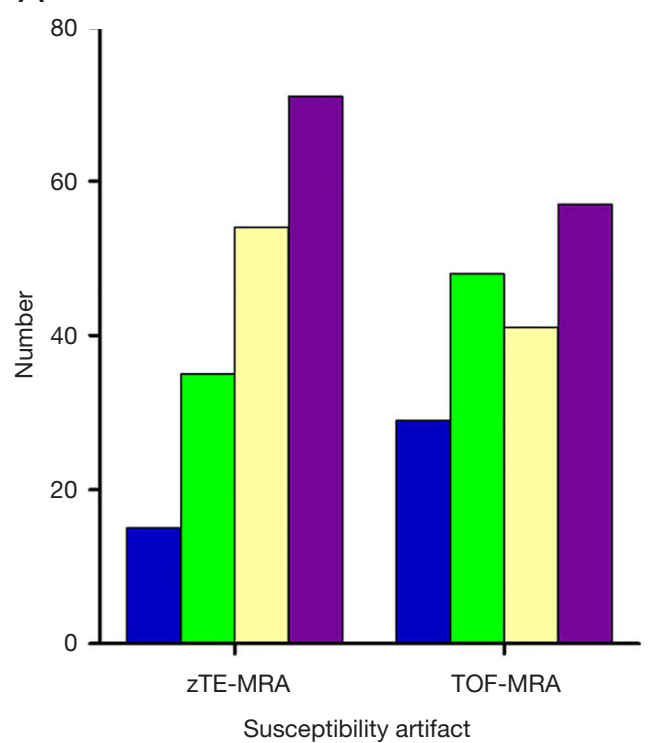

B
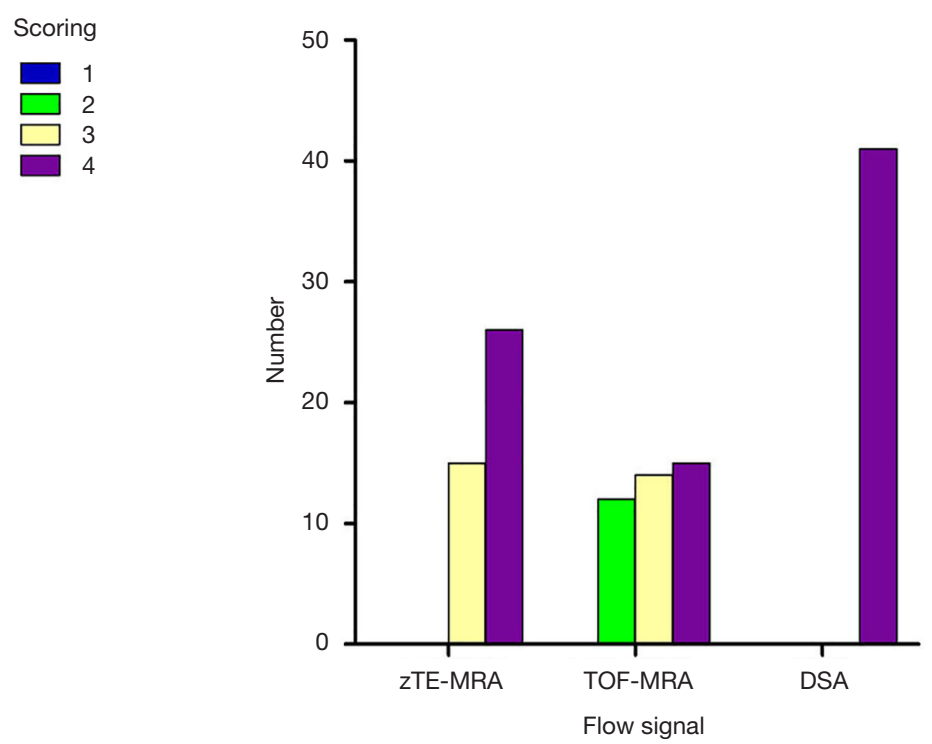

Figure 1 The score distribution of magnetic susceptibility artifact and flow signal. (A) Histogram showing the distribution of different scores (blue, 1 point; green, 2 points; brown, 3 points; and purple, 4 points) for susceptibility artifact intensity of zTE- and TOF-MRA imaging of 175 patients with ICAD. (B) Histogram showing the distribution of different scores (blue, 1 point; green, 2 points; brown, 3 points; and purple, 4 points) for flow signal in the parent artery in 41 patients with ICAS, according to zTE-, TOF-, and DSA imaging, with DSA imaging serving as the reference. ICAD, intracranial atherosclerotic disease; ICAS, intracranial arterial stenosis.

software 18.0 (IBM, Chicago, IL). Weighted kappa (к) statistics were first used to assess inter-observer agreement for susceptibility artifact intensity and flow signal evaluation of zTE- and TOF-MRA images, as well as inter-reader agreement for stenosis measurement. The embedded Wilcoxon signed-rank test toolbox was then applied to evaluate the differences between zTE- and TOF-MRA images regarding susceptibility artifact intensity and flow signal in target artery. Diagnostic sensitivity, specificity and diagnostic accuracy were computed for zTE- and TOFMRA by receiver operating characteristic curve analysis. In addition, nonparametric test of multiple paired samples was employed to test inter-group difference of stenosis assessment among zTE-, TOF-MRA and DSA, $\mathrm{P}<0.05$ is considered a statistically significant difference. Finally, weighted kappa $(\kappa)$ statistics were employed to separately estimate the diagnostic consistency of the zTE- and TOFMRA methods with DSA. The significance threshold was set at $\mathrm{P}=0.05$.

\section{Results}

The total number of intracranial atherosclerotic lesion sites was 93 , which were distributed in the anterior, middle, and posterior cerebral arteries of the 41 patients who underwent DSA.

High inter-observer agreement (weighted $\kappa=0.78$ ) was found for susceptibility artifact intensity and flow signal evaluation of the zTE- and TOF-MRA images of all patients. Inter-reader agreement for DSA stenosis measurement was also obtained (weighted $\kappa=0.80$ ).

zTE-MRA images were significantly higher quality, in terms of susceptibility artifact, than TOF-MRA images in bilateral ICAs (mean score, $3.03 \pm 0.98$ vs. $2.72 \pm 1.09$; Wilcoxon rank test, $\mathrm{P}=0.017$; Figure $1 A$ ). Flow signals in bilateral ICAs were assessed in both zTE- and TOFMRA images using DSA as a reference. For the 41 patients also assessed using DSA, scores using zTE-MRA were significantly higher than those with TOF-MRA (mean: $3.63 \pm 0.49$ vs. $3.07 \pm 0.82 ; \mathrm{P}<0.001)$. The number of subjects with the highest scores using zTE-MRA and TOF were 26 and 15, respectively. The distribution of scores is shown in Figure $1 B$. It can also be found in Figure 2 that zTEMRA shows very good performance of susceptibility artifact signal quality/flow signal. In addition, zTE-MRA generated significantly higher scores of image quality than that of 

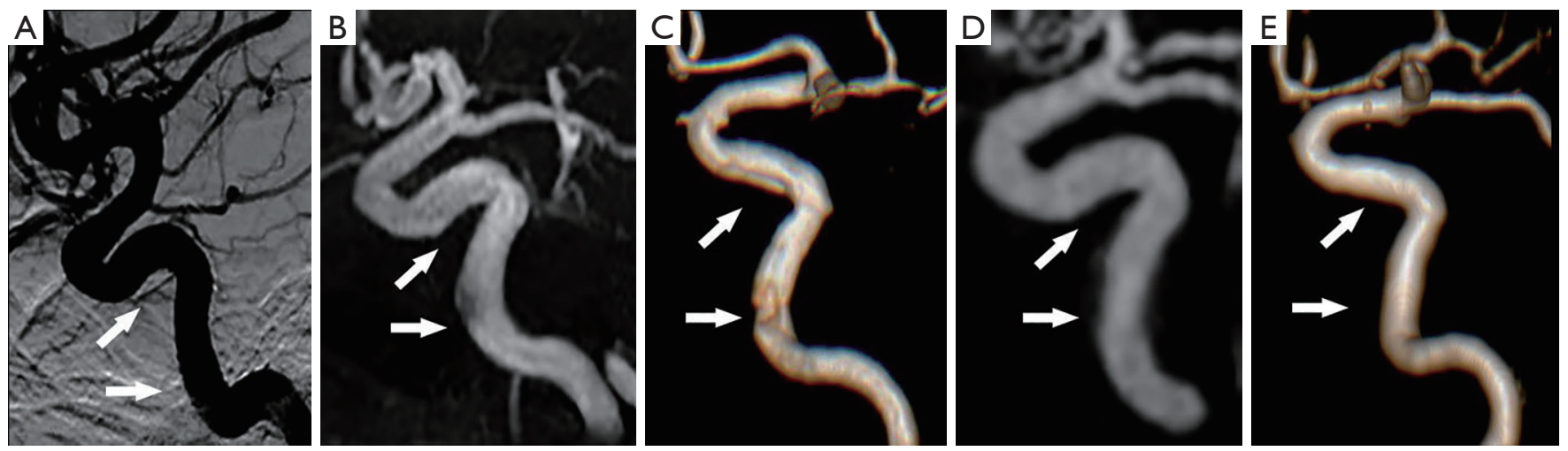

Figure 2 A 69-year-old male patient with a chief complaint of repeated headaches for 1 week. DSA (A) and TOF-MRA (B,C) imaging, with poor quality/flow signal scored as $3 / 3$ points. zTE-MRA imaging (D,E) showing excellent susceptibility artifact signal quality/flow signal scores (4/4 points). The arrows indicate regions of interest.

Table 1 Overview of TOF-, zTE-MRA and DSA for intracranial vascular assessment

\begin{tabular}{lccccccc}
\hline Image acquisition & Mild stenosis & Moderate stenosis & Severe stenosis & Total & Occlusion & No stenosis & Specificity/sensitivity \\
\hline TOF-MRA & 28 & 30 & 23 & 93 & 12 & 0 & $67 \% / 100 \%$ \\
zTE-MRA & 30 & 29 & 20 & 93 & 8 & 6 & $78 \% / 100 \%$ \\
DSA & 33 & 26 & 16 & 93 & 8 & 10 & N/A \\
\hline
\end{tabular}

Specificity/sensitivity represented the results for overall diagnosis of stenosis. zTE-MRA, zero echo time magnetic resonance angiography; DSA, digital subtraction angiography; N/A, not applicable.

TOF-MRA in both ROI of M1 and BA (Figure S1).

The intracranial vascular stenosis assessment of TOF, zTE-MRA and DSA were showed in Table 1. ZTEMRA had higher diagnostic specificity (78\% vs. 67\%) and accuracy (96\% vs. 94\%) than TOF-MRA, while the two methods had comparable diagnostic sensitivity (100\%). The stenosis degree was overestimated using TOF-MRA $(\mathrm{P}<0.05)$. In addition, weighted kappa $(\kappa)$ statistics analysis revealed high diagnostic consistency between zTE-MRA and DSA images for evaluating stenosis severity in patients with ICAS (weighted $\kappa=0.80$ ) (Figures 3,4).

\section{Discussion}

In this study, we investigated the feasibility of zTE-MRA for diagnosis of ICAD. By comparison with TOF-MRA, we found that zTE-MRA had more robust performance for imaging ICAD, including higher signal intensity with less artifact and stronger flow signal in the artery. Moreover, clinical findings using zTE-MRA were highly consistent with those using the reference DSA method for stenosis assessment in patients with ICAD.

In this study, zTE-MRA scored significantly higher than TOF-MRA for susceptibility artifact assessment. Susceptibility artifact is common in intracranial MRA, particularly in the distal ICA (28), and primarily associated with the vessels themselves, including distorted vascular shape, vessel wall calcification, and boundary interference between air-soft tissue and bone-soft tissue (29-31). As a conventional MRA technique, TOF-MRA is sensitive to susceptibility artifact $(28,32)$, and thus its application for patients with intracranial vascular diseases is generally challenging. As reported in previous studies, it is not possible to effectively visualize flow in an intracranial stent or on follow-up assessment of coiled aneurysms by TOFMRA $(15,16)$. In contrast, a novel feature of zTE-MRA is that it uses a $3 \mathrm{D}$ radial acquisition read-out scheme with an ultrashort echo time ( $10 \mu s)(33)$. Thus, zTE-MRA can generate high signal intensity and is immune to local field inhomogeneity and magnetic susceptibility artifacts, which is essential for accurate display of blood vessels (34).

In addition, relative to TOF-MRA, zTE-MRA showed 


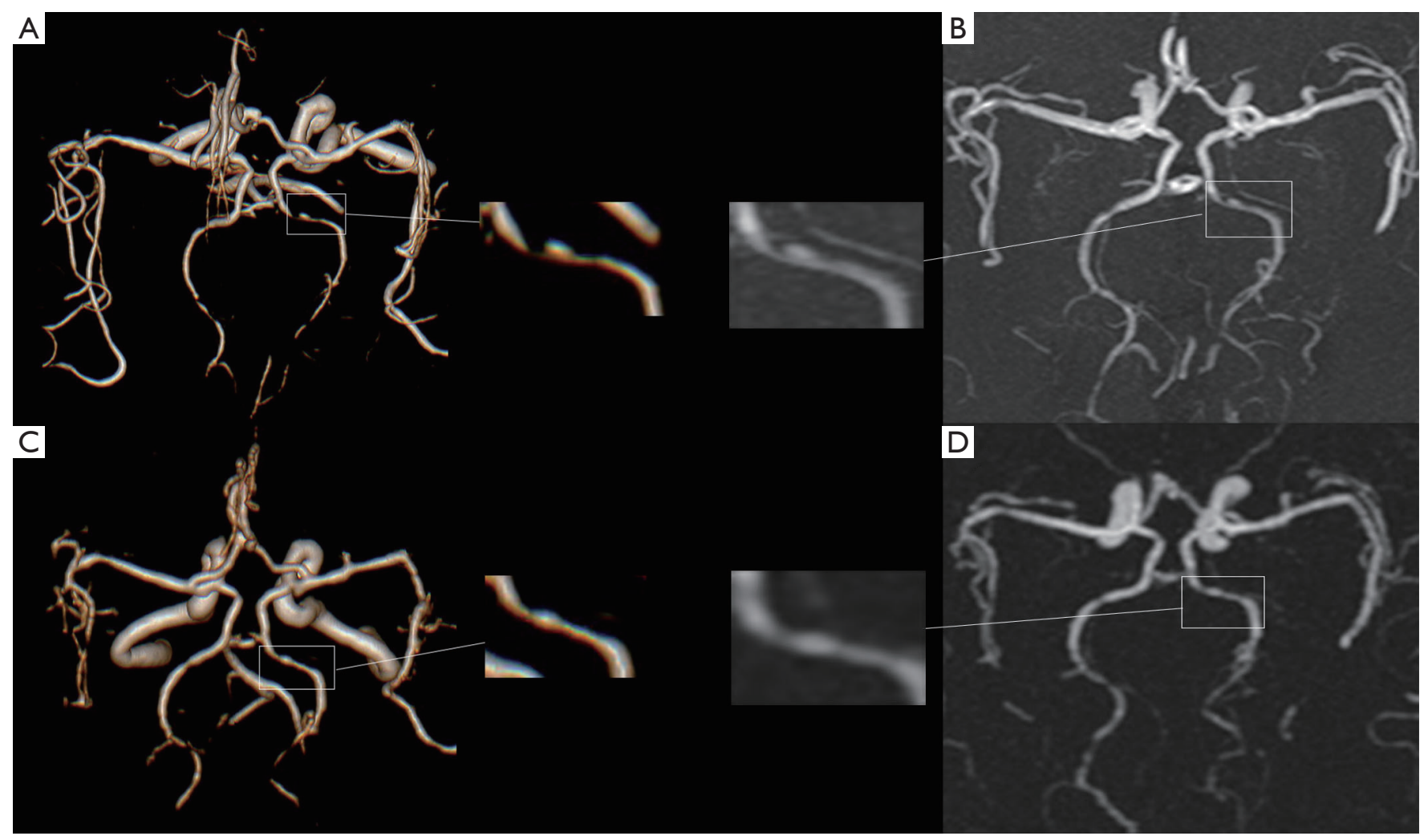

Figure 3 A 66-year-old male patient with a chief complaint of repeated dizziness and headaches for one week. TOF-MRA imaging (A,B) showed moderate stenosis of the right posterior cerebral artery (rectangular frame); however, zTE-MRA imaging (C,D) showed mild stenosis in the same artery (rectangular frame).

higher flow signals in the ICA in this study, and could thus be used to perform more accurate assessment of vascular stenosis. Flow disturbance mostly occurs in irregular and twisted blood vessels. For patients with ICAS, cerebral blood flow in the stenotic region is not regular in velocity or flow direction. Hence, the abnormal blood flow is often largely saturated by repeated RF pulses, resulting in low signal intensities on TOF-MRA $(11,29,32,35)$, which could explain why the severity of stenosis was overestimated by TOF-MRA in the present study. This finding is in agreement with previous reports that TOF-MRA is more likely to produce false negatives or false positives $(32,36)$. In comparison, zTE-MRA is a continuous ASL-based angiography method, which relies on the refreshment of blood flow in intracranial arteries with labeled blood flow from carotid arteries. Inflowing blood is magnetically labeled with an endogenous contrast tracer for vessel imaging; thus, no constraint is imposed on the direction of blood flow (33). Hence, zTE-MRA is not sensitive to turbulence or vascular distortion. In addition, because of the intrinsic ASL strategy used in zTE-MRA, the resulting images are generated via subtraction, to yield an angiographic image with almost zero background (15), further enhancing the flow signal contrast. The ASL based MRA technique is sensitive to slow flow velocity. Most vessels in the brain with normal flow velocity can reached with labeled blood within the labeling time assigned for zTE-MRA, however, vessels with significantly slow velocity may not be labeled, resulting in poor imaging. This could be the potential reason that posterior cerebral arteries are shown less on zTE-MRA compared to TOF-MRA.

In this study, we selected the intracranial segment of the ICA to analyze the susceptibility artifact and flow signal of zTE- and TOF-MRA images. The main reason for this choice is that this segment is essentially a twisted vessel with complicated blood flow, and has been reported to be the most vulnerable location for patients with ICAD $(37,38)$; therefore, the intracranial segment of the ICA 


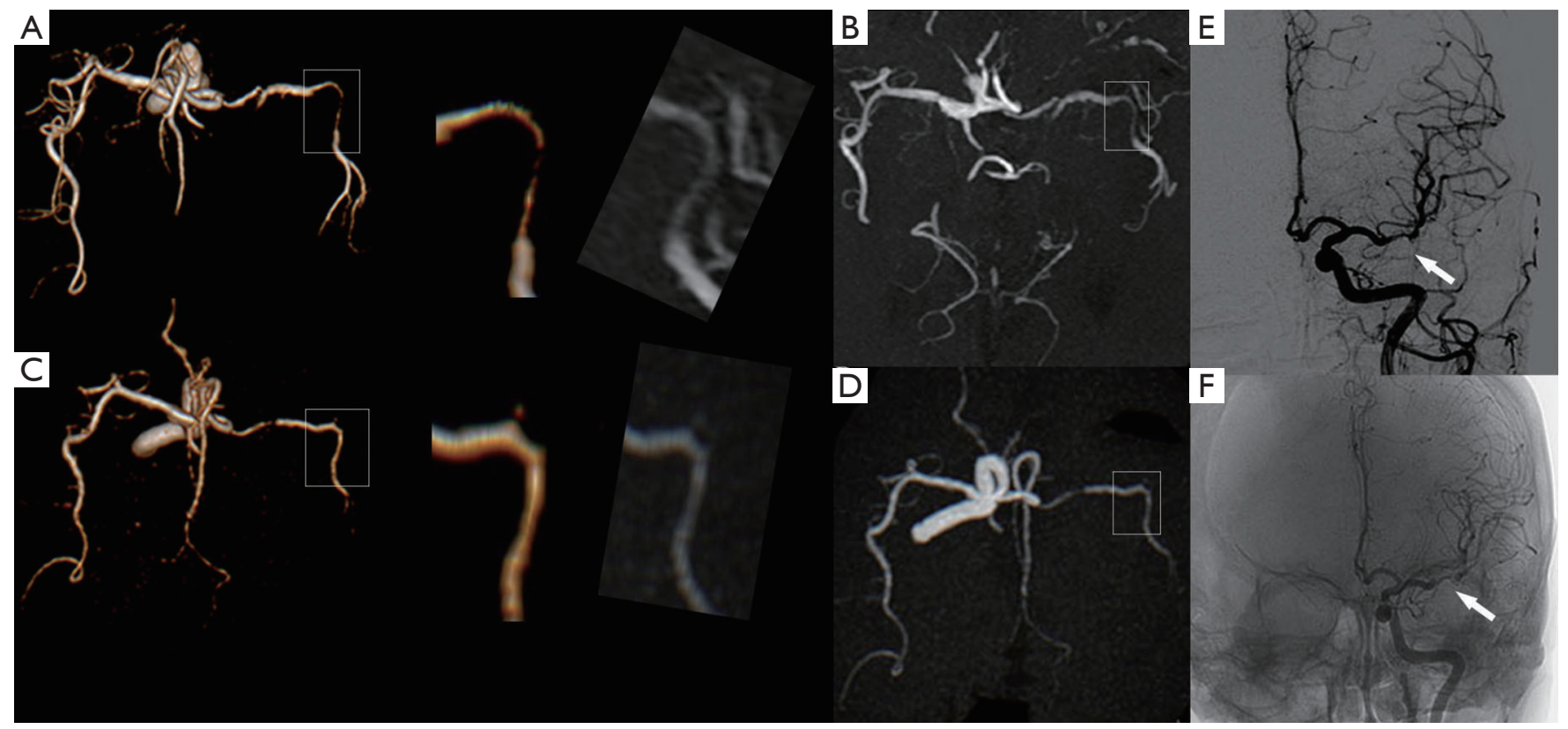

Figure 4 A 58-year-old male patient with a chief complaint of repeated headaches for 3 days. TOF-MRA imaging (A,B) showed moderate stenosis of the right mid cerebral artery (rectangular frame); however, zTE-MRA imaging (C,D) showed mild stenosis in the same artery (rectangular frame). 2D (E)- and 3D (F)-DSA imaging serving as the reference. The arrows indicate stenosis area of interest. D, dimensional.

was considered a representative ROI for assessment in this study. It should also be noted that this is a limitation of the present study, we did not perform evaluation on other intracranial vessel segments. Another limitation is that we did not measure the length of vascular stenosis, which can have an added in the evaluation of the stenosis level. In the follow up study, we will conduct a comprehensive analysis on intracranial arteries with more vessel segments as well as with the measurement of the stenosis length. At last, we have to admit that not identical image resolution was applied in zTE-MRA and TOF-MRA, which may have a potential impact on the fair comparison of image quality.

DSA remains a gold standard for clinical diagnoses of intracranial vascular diseases (7). zTE-MRA showed a more comparable assessment of flow signal than TOF-MRA to that achieved by DSA. Therefore, highly consistent clinical findings were obtained between zTE-MRA and DSA imaging for evaluation of stenosis severity in patients with ICAD. Although the stenosis overestimation in some cases was occurred relative to a gold standard DSA, zTE-MRA, compared with TOF-MRA, exhibited higher diagnostic specificity, and more accurately distinguished the degree of vascular stenosis in the anterior, middle, and posterior cerebral arteries. The assessment of BA stenosis was not included in this study, mainly due to the small number of BA stenosis. As reported previously, the total incidence of stenosis in the anterior, middle, and posterior cerebral arteries of Chinese Han people is significantly larger than that of BA (80\% vs. 5.2\%) (39). However, we admitted that it would be better to include BA for a more comprehensive vessel stenosis assessment between zTE- and TOR-MRA. This will be further implemented in our follow-up study.

Moreover, zTE-MRA can achieve silent intracranial angiography, due to the minor gradient adjustment required during image acquisition. This feature allows scanning in an almost silent environment allowing patients to feel more comfortable during assessment. This feature can largely avoid the degradation of image quality introduced by patient anxiety or involuntary movement. In addition, also because of the minor gradient adjustment, eddy current artifacts can be minimized in zTE-MRA imaging, further improving image quality.

TOF-MRA has achieved 2 minutes shorter scan time but higher image resolution than zTE-MRA in this study, and shorter scan time might also allow for less motion artifact from patients during measurement. However, higher image resolution in TOF-MRA didn't help improve image quality and showed relatively lower diagnostic accuracy 
for intracranial artery stenosis. In contrast, even though zTE-MRA measurement was a bit longer than TOF-MRA in this study, the quietness of zTE-MRA can reduce the motion risk of patients during measurement. In addition, zTE-MRA showed higher image quality and good ability to assess vascular stenosis with DSA. With these advantages, zTE-MRA might be considered with added clinical value in diagnosis of ICAS.

Notably, Shang et al. (23) recently published an interesting study that also confirmed the clinical value of zTE-MRA for imaging cerebrovascular diseases. Compared with their study, we used DSA as the reference for ICAD diagnosis, which we believe is more accurate than the CTA applied in their investigation (40). DSA can monitor contrast injection through a vessel in real time to accurately evaluate stenosis severity, and is thus often used in endovascular treatment for acute cerebral embolism, which cannot be achieved by CTA (41). Furthermore, the present sample size is larger, and the recruited patients were only those with ICAS. These features of our study may mean that the results reported here are more broadly representative.

Similar to previous reports $(23,42)$, we also found that zTE-MRA resulted in lower quality imaging of distal small branched vessels than TOF-MRA (Figure 4). In essential, zTE-MRA is a continuous ASL-based angiography method (15). The vessel signal is obtained by subtracting labeled with unlabeled arterial blood signal. Distal small vessels are however not a good candidate for imaging using zTE-MRA, as the blood flow requests relatively long time to reach distal vessels and the correspondingly labeled proton signal is largely recovered due to T1 relaxation effect. The resultant subtracted signal between labeled and non-labeled flow is thus decreased, leading to less qualified vessel imaging. However, this may not be crucial for ICAD diagnosis, as ICAD is more likely to be found in the middle-sized and larger branches $(43,44)$. Further, there are many other techniques available for distal vessel imaging in the clinic. Obara et al. (45) used accelerationselective ASL MRA to achieve robust visualization of distal cerebral arteries and collateral vessels. In addition, one other study also obtained satisfactory imaging of collateral circulation, based on a four-dimensional (4D)-ASL-MRA technique (46).

\section{Conclusions}

In conclusion, in the present study we found that, relative to TOF-MRA, zTE-MRA has higher image quality, less sensitivity to abnormal blood flow at different velocities in ICA. Besides, it also has excellent diagnostic consistency for assessment of stenosis severity, compared with DSA. Due to its superior performance for ICAD imaging, the zTE-MRA technique might thus be suggested as an alternative method for application in patients with ICAD in the clinic.

\section{Acknowledgments}

The authors thank the patients who participated in this study and the staff of the Department of Radiology for various types of support.

Funding: This study was supported by the Doctor of Entrepreneurship and Innovation in Jiangsu Province, China (No. 2019204006).

\section{Footnote}

Conflicts of Interest: All authors have completed the ICMJE uniform disclosure form (available at http://dx.doi. org/10.21037/qims-20-696). The authors have no conflicts of interest to declare.

Ethical Statement: The study was approved by local ethical committee of Affiliated Hospital of Xuzhou Medical University (No.: XYFY2018-JS012-01) and written informed consent was obtained from the patient for publication of this study and any accompanying images. A copy of the written consent is available for review by the Editor-in-Chief of this journal.

Open Access Statement: This is an Open Access article distributed in accordance with the Creative Commons Attribution-NonCommercial-NoDerivs 4.0 International License (CC BY-NC-ND 4.0), which permits the noncommercial replication and distribution of the article with the strict proviso that no changes or edits are made and the original work is properly cited (including links to both the formal publication through the relevant DOI and the license). See: https://creativecommons.org/licenses/by-nc-nd/4.0/.

\section{References}

1. Banerjee C, Chimowitz MI. Stroke Caused by Atherosclerosis of the Major Intracranial Arteries. Circ Res 2017;120:502-13.

2. Battistella V, Elkind M. Intracranial atherosclerotic disease. 
Eur J Neurol 2014;21:956-62.

3. Gorelick PB, Wong KS, Bae HJ, Pandey DK. Large artery intracranial occlusive disease: a large worldwide burden but a relatively neglected frontier. Stroke 2008;39:2396-9.

4. Holmstedt CA, Turan TN, Chimowitz MI. Atherosclerotic intracranial arterial stenosis: risk factors, diagnosis, and treatment. Lancet Neurol 2013;12:1106-14.

5. Ren C, Yuan J, Tong S, Xue Y, Wu H, Li W, Wang J, Sun Z, Gong L, Wang X, Liu J, Chen Q, Liu H. Memory Impairment Due to a Small Acute Infarction of the Columns of the Fornix. J Stroke Cerebrovasc Dis 2018;27:e138-e143.

6. Bash S, Villablanca JP, Jahan R, Duckwiler G, Tillis M, Kidwell C, Saver J, Sayre J. Intracranial vascular stenosis and occlusive disease: evaluation with CT angiography, MR angiography, and digital subtraction angiography. AJNR Am J Neuroradiol 2005;26:1012-21.

7. Feldmann E, Wilterdink JL, Kosinski A, Lynn M, Chimowitz MI, Sarafin J, Smith HH, Nichols F, Rogg J, Cloft HJ, Wechsler L, Saver J, Levine SR, Tegeler C, Adams R, Sloan M, Stroke O, Neuroimaging of Intracranial Atherosclerosis Trial I. The Stroke Outcomes and Neuroimaging of Intracranial Atherosclerosis (SONIA) trial. Neurology 2007;68:2099-106.

8. Wang J, Li R, Liu M, Nie Z, Jin L, Lu Z, Li Y. Impaired cerebral hemodynamics in late-onset depression: computed tomography angiography, computed tomography perfusion, and magnetic resonance imaging evaluation. Quant Imaging Med Surg 2020;10:1763-74.

9. Arias EJ, Vajapey S, Reynolds MR, Chicoine MR, Rich KM, Dacey RG Jr, Dorward IG, Derdeyn CP, Moran CJ, Cross DT, 3rd, Zipfel GJ, Dhar R. Utility of Screening for Cerebral Vasospasm Using Digital Subtraction Angiography. Stroke 2015;46:3137-41.

10. Mocco J, Fargen KM, Albuquerque FC, Bendok BR, Boulos AS, Carpenter JS, Fiorella DJ, Hoh BL, Howington JU, Liebman KM, Natarajan SK, Rai AT, RodriguezMercado R, Siddiqui AH, Snyder KV, Veznedaroglu E, Hopkins LN, Levy EI. Delayed thrombosis or stenosis following enterprise-assisted stent-coiling: is it safe? Midterm results of the interstate collaboration of enterprise stent coiling. Neurosurgery 2011;69:908-13; discussion 913-4.

11. Cirillo M, Scomazzoni F, Cirillo L, Cadioli M, Simionato F, Iadanza A, Kirchin M, Righi C, Anzalone N. Comparison of 3D TOF-MRA and 3D CE-MRA at 3T for imaging of intracranial aneurysms. Eur J Radiol 2013;82:e853-9.

12. Carvalho M, Oliveira A, Azevedo E, Bastos-Leite AJ.
Intracranial arterial stenosis. J Stroke Cerebrovasc Dis 2014;23:599-609.

13. Choi CG, Lee DH, Lee JH, Pyun HW, Kang DW, Kwon SU, Kim JK, Kim SJ, Suh DC. Detection of intracranial atherosclerotic steno-occlusive disease with 3D time-offlight magnetic resonance angiography with sensitivity encoding at 3T. AJNR Am J Neuroradiol 2007;28:439-46.

14. Cho WS, Kim SS, Lee SJ, Kim SH. The effectiveness of $3 \mathrm{~T}$ time-of-flight magnetic resonance angiography for follow-up evaluations after the stent-assisted coil embolization of cerebral aneurysms. Acta Radiol 2014;55:604-13.

15. Irie R, Suzuki $M$, Yamamoto $M$, Takano N, Suga $Y$, Hori M, Kamagata K, Takayama M, Yoshida M, Sato S, Hamasaki N, Oishi H, Aoki S. Assessing Blood Flow in an Intracranial Stent: A Feasibility Study of MR Angiography Using a Silent Scan after Stent-Assisted Coil Embolization for Anterior Circulation Aneurysms. AJNR Am J Neuroradiol 2015;36:967-70.

16. Shang S, Ye J, Luo X, Qu J, Zhen Y, Wu J. Follow-up assessment of coiled intracranial aneurysms using zTE MRA as compared with TOF MRA: a preliminary image quality study. Eur Radiol 2017;27:4271-80.

17. Song Y, Qi P, Huang J, Jiao S, Zhang J, Wang D, Chen $M$. Application of zero echo time MR angiography in follow-up of intracranial aneurysm remnant and in-stent lumen after embolization: a comparison study with digital subtraction angiography. Acta Radiol 2020;61:480-6.

18. Takano N, Suzuki $M$, Irie R, Yamamoto $M$, Teranishi K, Yatomi K, Hamasaki N, Kumamaru KK, Hori M, Oishi H, Aoki S. Non-Contrast-Enhanced Silent Scan MR Angiography of Intracranial Anterior Circulation Aneurysms Treated with a Low-Profile Visualized Intraluminal Support Device. AJNR Am J Neuroradiol 2017;38:1610-6.

19. Katsuki M, Narita N, Ozaki D, Sato Y, Iwata S, Tominaga T. Three tesla magnetic resonance angiography with ultrashort echo time describes the arteries near the cerebral aneurysm with clip and the peripheral cerebral arteries. Surg Neurol Int 2020;11:224.

20. Tomura N, Kokubun M, Horiuchi K, Watanabe Z. Comparison of TOF-MRA and silent scan-MRA in depicting cerebral arteries in patients with Moyamoya disease. Acta Radiol 2019;60:1321-8.

21. Arai N, Akiyama T, Fujiwara K, Koike K, Takahashi S, Horiguchi T, Jinzaki M, Yoshida K. Silent MRA: arterial spin labeling magnetic resonant angiography with ultra-short time echo assessing cerebral arteriovenous 
malformation. Neuroradiology 2020;62:455-61.

22. Moon JI, Baek HJ, Ryu KH, Park H. A novel noncontrast-enhanced MRA using silent scan for evaluation of brain arteriovenous malformation: A case report and review of literature. Medicine (Baltimore) 2017;96:e8616.

23. Shang S, Ye J, Dou W, Luo X, Qu J, Zhu Q, Zhang H, Wu J. Validation of Zero TE-MRA in the Characterization of Cerebrovascular Diseases: A Feasibility Study. AJNR Am J Neuroradiol 2019;40:1484-90.

24. Wu J. Promote recognition technology of cerebral atherosclerosis in medical institutions at different levels, standardized the diagnosis: interpretation of "criteria for the screening and diagnosis of cerebral atherosclerosis (2014)". Zhonghua Yi Xue Za Zhi 2014;94:3697-8.

25. Madio DP, Lowe IJ. Ultra-fast imaging using low flip angles and FIDs. Magn Reson Med 1995;34:525-9.

26. Takano N, Suzuki M, Irie R, Yamamoto M, Hamasaki N, Kamagata K, Kumamaru KK, Hori M, Oishi H, Aoki S. Usefulness of Non-Contrast-Enhanced MR Angiography Using a Silent Scan for Follow-Up after Y-Configuration Stent-Assisted Coil Embolization for Basilar Tip Aneurysms. AJNR Am J Neuroradiol 2017;38:577-81.

27. Oishi H, Fujii T, Suzuki M, Takano N, Teranishi K, Yatomi K, Kitamura T, Yamamoto M, Aoki S, Arai H. Usefulness of Silent MR Angiography for Intracranial Aneurysms Treated with a Flow-Diverter Device. AJNR Am J Neuroradiol 2019;40:808-14.

28. Bos D, Portegies ML, van der Lugt A, Bos MJ, Koudstaal PJ, Hofman A, Krestin GP, Franco OH, Vernooij MW, Ikram MA. Intracranial carotid artery atherosclerosis and the risk of stroke in whites: the Rotterdam Study. JAMA Neurol 2014;71:405-11.

29. Kaufmann TJ, Kallmes DF. Utility of MRA and CTA in the evaluation of carotid occlusive disease. Semin Vasc Surg 2005;18:75-82.

30. McCoy MR, Broussalis E, Killer M, Steinbacher J, Klausner F. Extensive pneumatized air cells causing susceptibility artifacts in the petrosus part of the ICA. Clin Neuroradiol 2017;27:91-6.

31. Deutschmann HA, Augustin M, Simbrunner J, Unger B, Schoellnast H, Fritz GA, Klein GE. Diagnostic accuracy of 3D time-of-flight MR angiography compared with digital subtraction angiography for follow-up of coiled intracranial aneurysms: influence of aneurysm size. AJNR Am J Neuroradiol 2007;28:628-34.

32. Miki H. [Pitfalls in diagnosing cerebrovascular diseases using magnetic resonance angiography]. Brain Nerve 2010;62:477-88.
33. Wu H, Block WF, Turski PA, Mistretta CA, Johnson KM. Noncontrast-enhanced three-dimensional (3D) intracranial MR angiography using pseudocontinuous arterial spin labeling and accelerated 3D radial acquisition. Magn Reson Med 2013;69:708-15.

34. Weiger M, Brunner DO, Dietrich BE, Muller CF, Pruessmann KP. ZTE imaging in humans. Magn Reson Med 2013;70:328-32.

35. Alibek S, Vogel M, Sun W, Winkler D, Baker CA, Burke $\mathrm{M}$, Gloger H. Acoustic noise reduction in MRI using Silent Scan: an initial experience. Diagn Interv Radiol 2014;20:360-3.

36. Townsend TC, Saloner D, Pan XM, Rapp JH. Contrast material-enhanced MRA overestimates severity of carotid stenosis, compared with 3D time-of-flight MRA. J Vasc Surg 2003;38:36-40.

37. Sanders-Taylor C, Kurbanov A, Cebula H, Leach JL, Zuccarello M, Keller JT. The carotid siphon: a historic radiographic sign, not an anatomic classification. World Neurosurg 2014;82:423-7.

38. Wei LM, Zhu YQ, Bao YQ, Lu HT, Zhang PL, Zhao $\mathrm{YW}, \mathrm{Li}$ M, Zhao JG. Atherosclerosis in intracranial or extracranial vessels in diabetic patients and the association with stroke subtype. Quant Imaging Med Surg 2019;9:960-67.

39. Zhou F, Tang J, Li P, Liao B, Qin C. Distribution of cerebral artery stenosis and risk factors in ethnic Zhuang and Han patients with ischemic stroke in Guangxi province. Ann Palliat Med 2020;9:256-63.

40. Prestigiacomo CJ, Sabit A, He W, Jethwa P, Gandhi C, Russin J. Three dimensional CT angiography versus digital subtraction angiography in the detection of intracranial aneurysms in subarachnoid hemorrhage. J Neurointerv Surg 2010;2:385-9.

41. Perry JT, Statler JD. Advances in vascular imaging. Surg Clin North Am 2007;87:975-93, vii.

42. Holdsworth SJ, Macpherson SJ, Yeom KW, Wintermark M, Zaharchuk G. Clinical Evaluation of Silent T1Weighted MRI and Silent MR Angiography of the Brain. AJR Am J Roentgenol 2018;210:404-11.

43. Huang YN, Gao S, Li SW, Huang Y, Li JF, Wong KS, Kay R. Vascular lesions in Chinese patients with transient ischemic attacks. Neurology 1997;48:524-5.

44. Feldmann E, Daneault N, Kwan E, Ho KJ, Pessin MS, Langenberg P, Caplan LR. Chinese-white differences in the distribution of occlusive cerebrovascular disease. Neurology 1990;40:1541-5.

45. Obara M, Togao O, Yoneyama M, Okuaki T, Shibukawa 
S, Honda H, Van Cauteren M. Acceleration-selective arterial spin labeling for intracranial MR angiography with improved visualization of cortical arteries and suppression of cortical veins. Magn Reson Med 2017;77:1996-2004.

46. Iryo Y, Hirai T, Nakamura $M$, Inoue $Y$, Watanabe $M$,

Cite this article as: Zhang C, Dou W, Yu K, Ji Y, Wang W, Sami MU, Shen Y, Xu K. The feasibility of non-contrastenhanced zero echo time magnetic resonance angiography for characterization of intracranial atherosclerotic disease. Quant Imaging Med Surg 2021;11(6):2442-2452. doi: 10.21037/qims20-696
Ando Y, Azuma M, Nishimura S, Shigematsu Y, Kitajima $M$, Yamashita Y. Collateral circulation via the circle of Willis in patients with carotid artery steno-occlusive disease: evaluation on 3-T 4D MRA using arterial spin labelling. Clin Radiol 2015;70:960-5. 


\section{Image quality comparison between TOF-MRA and zTE-MRA}

A Flow signal scoring of Middle cerebral arteries (M1)

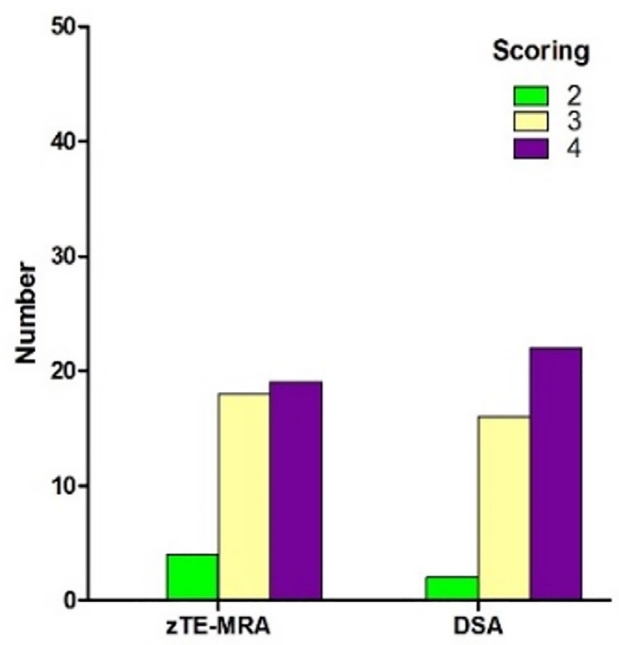

C

Susceptibility artifact scoring of Middle cerebral arteries (M1)

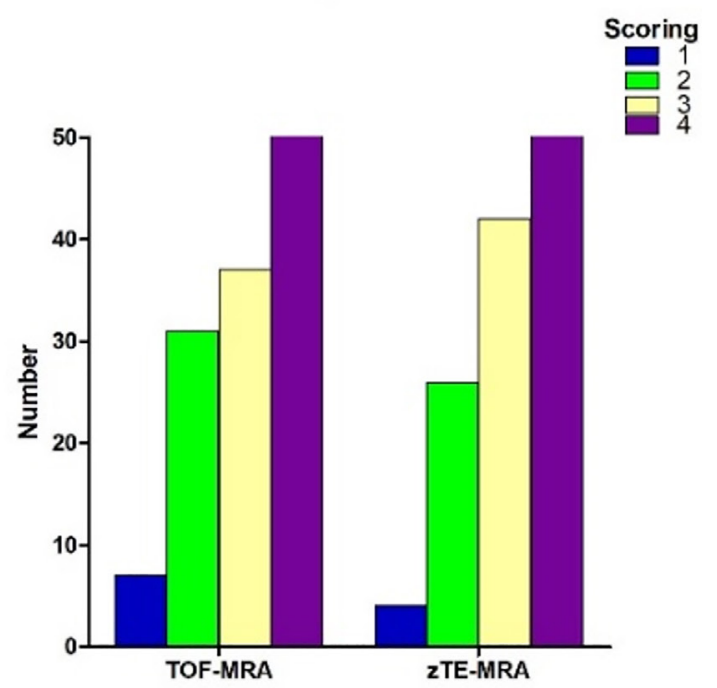

\section{B Flow signal scoring of Basilar artery}

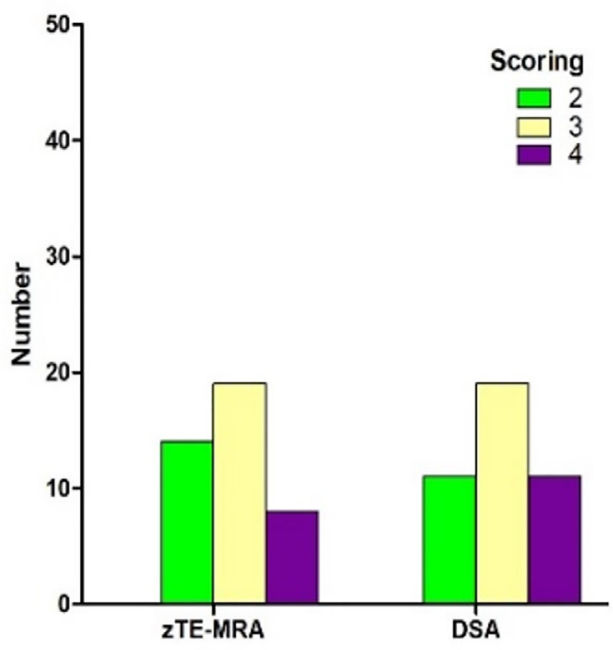

D

Susceptibility artifact scoring of Basilar artery

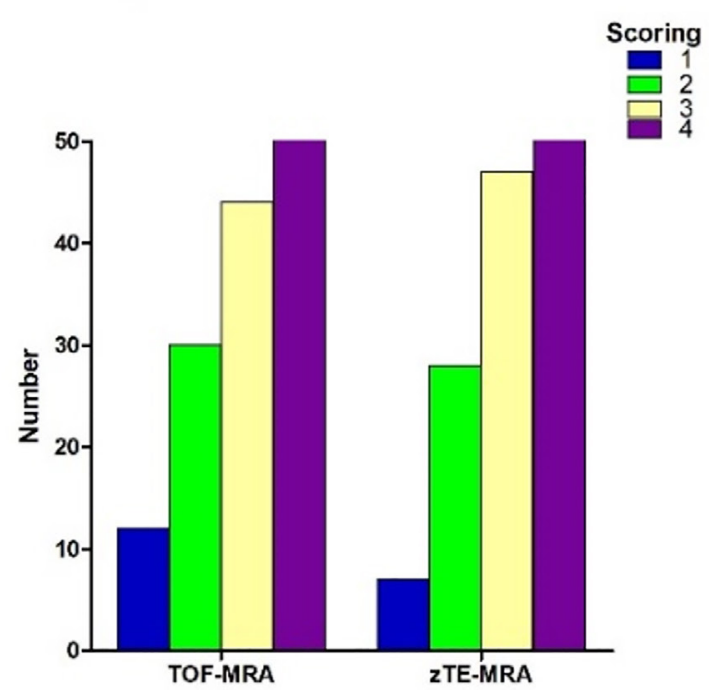

Figure S1 As shown in the following figure, in middle cerebral arteries (MCA) M1 and basilar artery (BA) regions, zTE-MRA showed significantly higher imaging quality than TOF-MRA images in terms of susceptibility artifact (M1: mean score, $3.39 \pm 0.82$ vs. $3.31 \pm 0.90$, Wilcoxon rank test, $\mathrm{P}<0.001$, Figure $S 1 A$ and, BA: mean score, $3.29 \pm 0.87$ vs. 3.20 \pm 0.95 , Wilcoxon rank test, $\mathrm{P}<0.001$, Figure S1B). Moreover, flow signal scores in zTE-MRA were also significantly higher than those in TOF-MRA in M1 (mean score, $3.68 \pm 0.47$ vs. 3.58 \pm 0.49 ; Wilcoxon rank test, $\mathrm{P}=0.046$, Figure $S 1 C$ ) and in $\mathrm{BA}$ (mean score, $3.68 \pm 0.47$ vs. 3.56 \pm .54 ; Wilcoxon rank test, $\mathrm{P}=0.025$, Figure S1D). 